\title{
Use of Euler free surface flow analysis to provide appropriate injection conditions in Euler-Lagrange numerical models
}

\author{
A. Rossetti, P. S. Nagabhushan, B. Barabas, J. P. Schnitzler, \\ A. Kefalas, F. K. Benra \& H. J. Dohmen \\ Chair of Turbomachinery, University of Duisburg-Essen, Germany
}

\begin{abstract}
The process of water injection at operating conditions of real gas turbines is an obscure two phase flow. This involves not only heat transfer and mass transfer but also the breakup and coalescence of droplets. To investigate such flows along with experiments, advanced CFD methods are also necessary. The EulerLagrange approach is one of the extensively used approaches for this process. However, providing a necessary boundary condition for this approach is quite challenging. In this paper, a Euler two phase free surface flow analysis in combination with a Linearized Instability Sheet Atomization (LISA) model is proposed to obtain the required injection conditions. This model considers the internal geometry of the nozzle and the air surrounding the outlet. The resulting free surface flow field is used to set the particle injection position and direction. Using these injection conditions a Euler-Lagrange analysis is set up. The EulerLagrange analysis results are analyzed and compared with the experimental data of water injection obtained from a hot air test rig.
\end{abstract}

Keywords: CFD, multiphase, atomization, LISA, free surface, VOF, EulerLagrange, water injection, high temperature, high pressure.

\section{Introduction}

Water injection into high speed airflow has been the subject of investigation for many years. Especially in gas turbines, wet compression or inlet fogging has been the focus of research due to its simplicity and cost-effectiveness to augment power and increase thermal efficiency $[1,2]$. 
The process of water injection and evaporation at high temperature and pressure is an intricate two phase flow. A thorough investigation of this process is needed to understand the behaviour of the droplets in such conditions. To accomplish this along with expensive experimental tests, investigations using CFD methods are necessary. Nevertheless, the presence of complex processes like evaporation, heat transfer, atomization, swirling flows etc. in such conditions present enough challenges for the present day commercial CFD tools.

Euler-Lagrange approach is one of the numerical approaches available to study these phenomena. However, providing necessary spray boundary conditions for this approach is still a challenge because of its dependency on the injection conditions. Injection conditions like droplet diameter, velocity and spray angle are of utmost importance for spray simulations [3, 4]. Many atomization studies have shown that the droplet diameter distribution is directly related to the film thickness [5]. Furthermore, the particle injection direction and velocity could be strongly affected by the presence of an external airflow.

The main objective of this paper is to present a procedure to set up a EulerLagrange analysis when the nozzle geometry and the operating condition are known. In the present work the droplet reference diameter, velocity and spray angle are obtained by analyzing the internal flow in a pressure swirl atomizer and combining it with an analytical breakup model.

Free surface or volume of fluid (VOF) technique has been employed by many researchers to investigate the internal flow behaviour of pressure swirl atomizers [6-8]. It was also found that VOF technique could capture the internal and external atomization with considerable agreement [8]. Due to the above mentioned capabilities, free surface technique has been used to obtain the water film characterization outside the nozzle. Whereas, the droplet diameter is computed using LISA model proposed by Senecal et al. [9], the input parameters for this model are obtained from the output of the free surface model. To demonstrate the accuracy of the numerical procedure, obtained results are compared qualitatively and quantitatively with the experimental data.

\section{Case of study}

Reference experimental data used in this paper are acquired using the experimental facility for investigating droplet evaporation at high temperature and pressure located at the University of Duisburg-Essen. It is designed to conduct laser based experiments with water sprays up to main flow boundary conditions of $1 \mathrm{MPa}$ and $648 \mathrm{~K}$. The conditions at which experiment were conducted are tabulated in table 1.

The main components of the testrig are shown in Figure 1. The measuring section consists of a glass tube $(\mathrm{d}=102 \mathrm{~mm}$ and $\mathrm{L}=1000 \mathrm{~mm})$ and water injection nozzle arrangement. Water is atomized inside the measuring section using a nozzle coaxial to the main air flow. A PDPA system with water cooled argon-ion laser and 3D traverse system is used to measure the spray. A detailed description of the PDPA system can be found in the investigations of Schnitzler et al. [10] and Kefalas et al. [11]. 


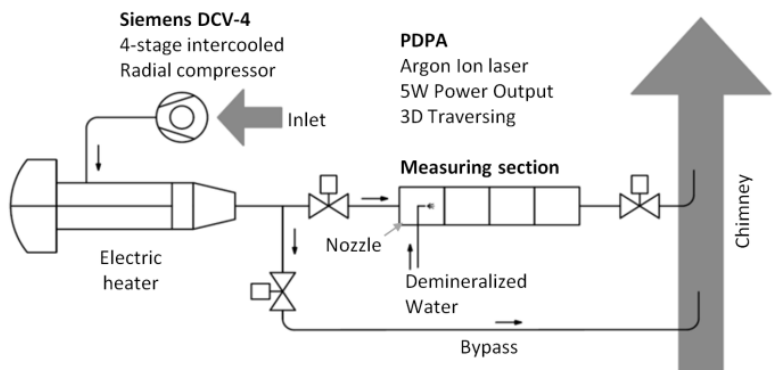

Figure 1: $\quad$ Experimental test rig.

Table 1: $\quad$ Experimental conditions.

\begin{tabular}{|l|c|l|}
\hline Boundary & Value & Units \\
\hline Air mass flow rate & 1.62 & {$[\mathrm{~kg} / \mathrm{s}]$} \\
\hline Air temperature & 465 & {$[\mathrm{~K}]$} \\
\hline Air pressure & 4.06 & {$[\mathrm{bar}]$} \\
\hline Water mass flow rate & 27.2 & {$[\mathrm{~g} / \mathrm{s}]$} \\
\hline Water temperature & 285 & {$[\mathrm{~K}]$} \\
\hline
\end{tabular}

\section{Numerical procedure}

The present paper describes the combined use of different numerical approaches to analyze the water injection into high velocity, pressurized and high temperature air flow.

The adopted procedure is shown in Figure 2 where the main features of each stage are presented. At first, a Euler Free Surface (EFS) analysis is used to model the water flow inside the nozzle and the air volume close to the nozzle exit up to the breakup. Further breakup is then modelled using an analytical model proposed by Senecal et al. [9]. The water sheet thickness, velocity and its trajectories obtained from the EFS model are used as inputs for the analytical breakup model. The Euler - Lagrange model is then set by taking the advantage of the information obtained by the two previous models to set proper injection conditions for the discrete phase.

CFD analyses are carried out using ANSYS CFX v.14. Each of these models will be presented and discussed in terms of set-up, results and their relation with each-other in the following sections. Finally to demonstrate the accuracy of the results, results obtained by Euler-Lagrange analyses are compared with the experimental results.

\section{Euler free surface model}

The main objective of this model is to describe the flow inside the nozzle and the water sheet development at the vicinity of the nozzle accounting the effects of the air flow field. The numerical model and the grid are shown in Figure 3. All 
the volumes are meshed using the structured grids. Refinements are set near wall to improve the grid resolution. The volume near an air-water interface is refined using 1:3 ratio to reduce the average cell size (Figure 3C). Grid size and cell dimensions are presented in table 2 .

Table 2: $\quad$ EFS grid features.

\begin{tabular}{|l|c|l|}
\hline Internal Domain & & \\
\hline Nodes & 1954317 & \\
\hline Elements & 1879586 & \\
\hline Average cell size & 120 & {$[\mu \mathrm{m}]$} \\
\hline Near wall element height & 4.0 & {$[\mu \mathrm{m}]$} \\
\hline External Domain & & \\
\hline Nodes & 467408 & \\
\hline Elements & 233244 & \\
\hline Average cell size & 700 & {$[\mu \mathrm{m}]$} \\
\hline Average cell size near the water surface & 140 & {$[\mu \mathrm{m}]$} \\
\hline
\end{tabular}

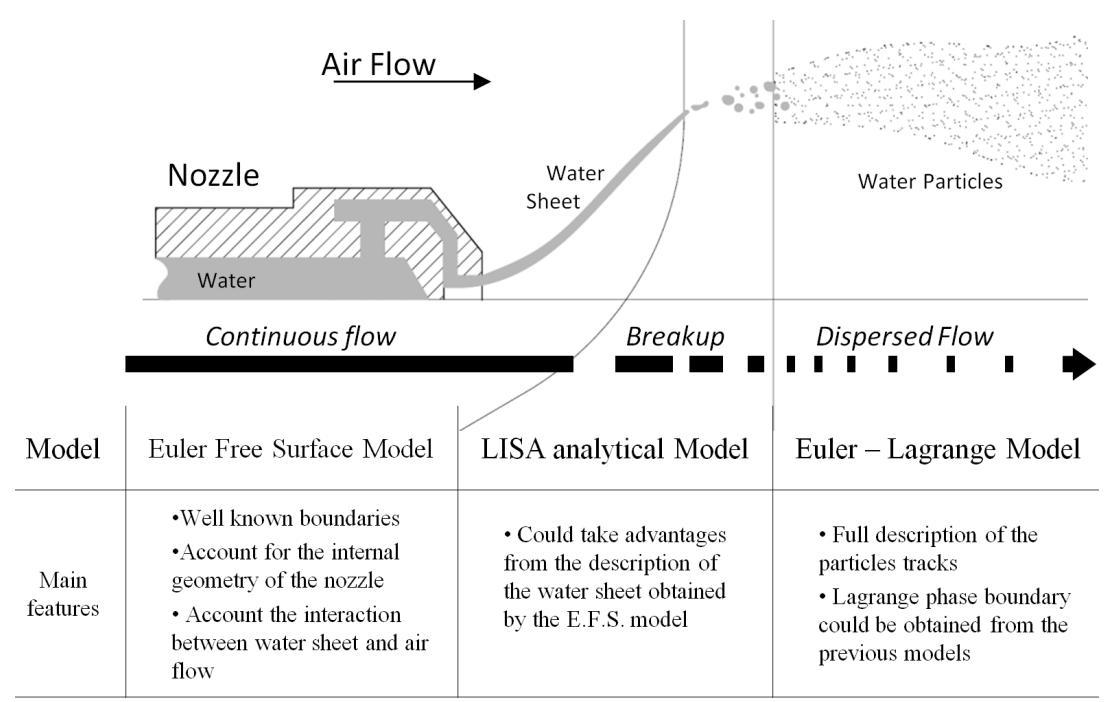

Figure 2: Numerical procedure main features.

The numerical model is divided into two domains. The volume inside the nozzle is defined by taking an advantage of the geometrical periodicity of the nozzle, thereby considering only $180^{\circ}$ and bounding it with periodicity conditions.

According to the numerical model of breakup mechanics proposed by Senecal et al. [9], the instability of the water sheet that lead to the detachment of the ligaments could be modelled as a two dimensional problem. According to this assumption the volume downstream of the swirl chamber and the external 
volume of the model are modelled as 2D one cell thick section under periodical condition. To match the pitch difference between the two domains, a stage type interface is used. This interface preserves the axial gradients while averaging the circumferential quantities. It also maintains the momentum equilibrium between its two sides, while the flow rate is scaled according to the pitch ratio between the two sides.

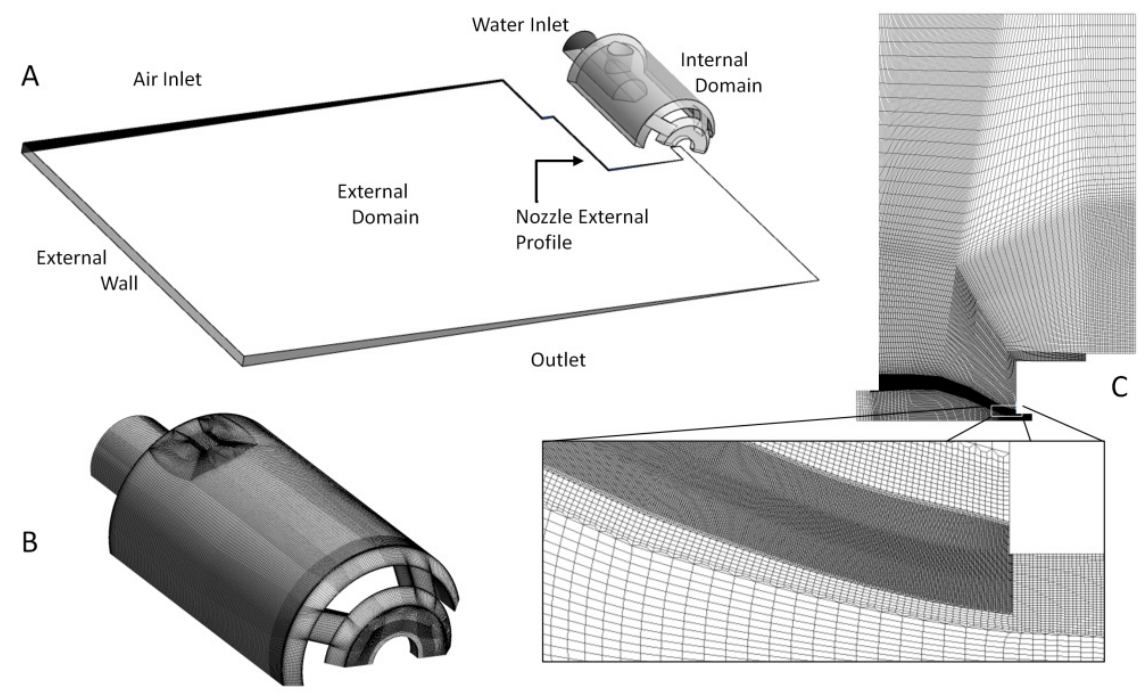

Figure 3: $\quad$ EFS computational domains and grids. (A) Entire computational domain. (B) Nozzle domain. (C) Cross sectional mesh and mesh refinements at the vicinity of the nozzle.

Water mass flow is set at the inlet of the nozzle domain, while air velocity is set at the inlet of the external domain. The outflow boundary is set imposing the average pressure on the section. Air is modelled as dry air ideal gas, while water is modelled using standard water with constant properties. Considering the presence of considerably high changes in the Reynolds number inside the nozzle, a two equation SST turbulence model is used. Surface tension coefficient between water and air surface is set to a value of $0.073\left[\mathrm{~N} \mathrm{~m}^{-1}\right]$ and the drag coefficient is set to 0.44 . Further details on the mathematical description of these parameters could found in the software documentation [12].

First of all a steady state simulation is performed. However, this run could not be considered to predict the waving of the water sheet which is intrinsically unsteady structure. This run is used only to solve the inner flow of the nozzle and a stable part of the water sheet discharged into the air. It is validated by comparing the total pressure behind the nozzle and the experimental data. The predicted value is $1.3 \%$ above the measured pressure, which is in accordance with the expected numerical accuracy.

To properly solve the external flow field near the nozzle, an unsteady state computation is performed. To reduce the computational time, the nozzle domain 
is discarded and an interface is substituted by the velocity profiles obtained in the steady run. Second order backward Euler scheme is used as a transient scheme. Relatively large time stepping is set at the beginning of the run to allow the flow field to develop independently from the initialization and then gradually reduce the time step. Initial time step is set to 1 [ $\mu \mathrm{s}]$ then reduced to $0.05[\mu \mathrm{s}]$ corresponding to an RMS and maximum Courant number respectively of 0.1 and 0.55 . In total $2860[\mu \mathrm{s}]$ are simulated resulting in 34250 time steps. Finest time step is used during the last $500[\mu \mathrm{s}]$.

\subsection{Model results}

EFS allows us to describe the flow inside the nozzle and the development of the water sheet at the vicinity of the nozzle. Due to the unsteadiness, the visualization of the water sheet development is done over 7 different times sampled using $6[\mu \mathrm{s}]$ time steps as shown in Figure 4.

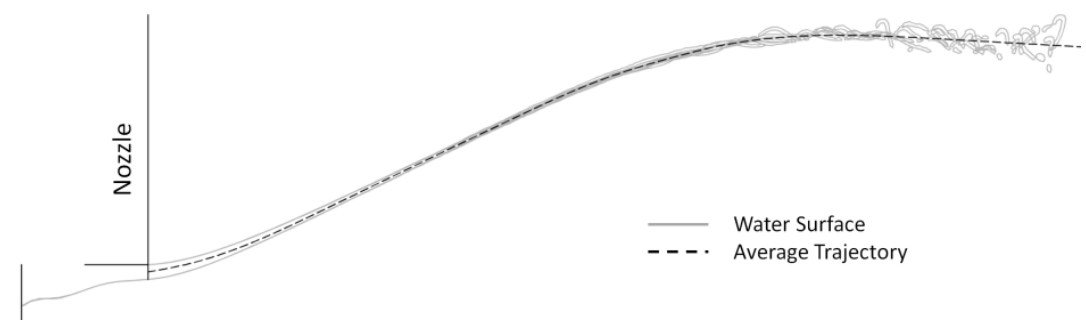

Figure 4: $\quad$ Water free surface and breakup.

As illustrated in Figure 4 the EFS model is able to capture some of the key features of the problem analyzed. Water exiting the nozzle bends outwards as a result of the spinning motion delivered by the nozzle. The conical trajectory is then perturbed by the upcoming air flow, which bends the water sheet towards the axis. The instability develops and grows along an average trajectory, finally leading to the breakup of the continuous sheet and the formation of the ligaments. It is important here to notice that the 2D nature of the analysis prevents further breakup of the ligaments into droplets. More details on the EFS results will be presented in the following sections.

\section{Breakup model}

The direct modelling of three dimensional breakup using a free surface model is challenging for most of the powerful computers, especially in industrial applications. Then the use of analytical correlation in this field is quite frequent. In this study, LISA model is used to study the primary breakup [9]. This model describes the breakup of a continuous liquid sheet moving in still air. After the sheet has left the nozzle, it experiences aerodynamic instabilities which break it into ligaments. Finally ligaments break into droplets as a result of the 
aerodynamic resistance. As the full description of this approach is described by Senecal et al. [9], only few important equations are described in this paper.

The water sheet response to an infinitesimal perturbation $\eta_{0}$ is modelled. The growth rate $\omega_{r}$ of this perturbation is a function of the wave number $k$ according to the following relation:

$$
\begin{gathered}
\omega_{r}(k, h)=-\frac{2 v_{l} k^{2} \tanh (k h)}{\tanh (k h)+Q}, \\
+\frac{\sqrt{4 v_{l} k^{2} \tanh (k h)^{2}-Q^{2} U^{2} k^{2}-[\tanh (k h)+Q]\left(-Q U^{2} k^{2}+\sigma k^{3} / \rho_{l}\right)}}{\tanh (k h)+Q}
\end{gathered}
$$

The breakup will occur when the perturbation $\eta_{0}$ grows up to the value $\eta_{b}$. Time, growth rate and amplitudes are related by the following:

$$
\int_{0}^{T} \omega_{r}(k, h) d \tau=\ln \left(\eta_{b} / \eta_{0}\right)
$$

where the quantity $\ln \left(\eta_{b} / \eta_{0}\right)$ is set to be 12 , based on the work of Dombrowski and Jones [13] and Dombrowski and Hooper [14].

The procedure proposed by Senecal et al. [9] is to maximize eqn (1) as a function of the wave number to obtain the highest growth rate $\Omega$ assuming constant half thickness of the water sheet. The critical time is computed using eqn (2). In the present case thickness is not constant. Critical conditions are then obtained by minimizing the time $T$ as a function of the growth rate using eqn (2), where the thickness was described as a function of time. For this purpose the description of the water trajectory is given as $h(t)=h_{0} r_{0} / r(t)$ according to the continuity equation. The average trajectory illustrated in Figure 4 is used to provide $r(t)$. The EFS results are also used to define the water velocity at the nozzle exit and the water thickness, avoiding the assumption of a discharge coefficient. The knowledge of the critical time $T$ and the wave number corresponding to the most critical waving frequency $K_{s}$ could be used to compute the breakup length $L_{b}=T U$, where $U$ is the total velocity at the nozzle exit. The diameter of ligament is then computed according to the continuity law:

$$
d_{L}=\sqrt{8 h / K_{s}}
$$

where two droplets are assumed to be formed by each wave (long wave assumption [9]). The size of the droplet formed by the ligament breakup is expressed as:

$$
\mathrm{d}_{\mathrm{rr}}=1.88 \mathrm{~d}_{\mathrm{L}}(1+30 \mathrm{Oh})^{\frac{1}{6}}
$$

where the particle Ohnesorge number is defined as, $O h=\mu /\left(\rho_{1} \sigma d_{1}\right)^{\frac{1}{2}}$ where both the density and the viscosity are referred to the liquid. The obtained diameter is used as the reference diameter in a Rosin-Rammler distribution function. Inputs and results of this model are presented in Table 3.

The result of this procedure could be validated by comparing the distribution obtained in the experimental data. Experimentally obtained reference diameter is $d_{r r E x p}=160[\mu \mathrm{m}]$, which is reasonably close to the predicted value of $d_{r r}=166$ $[\mu \mathrm{m}]$. 
Table 3: $\quad$ LISA setup and results.

\begin{tabular}{|l|c|l|}
\hline Input & & \\
\hline$V_{l}$ & 27.5 & {$[\mathrm{~m} / \mathrm{s}]$} \\
\hline$h_{0}$ & 112 & {$[\mu \mathrm{m}]$} \\
\hline Output & & \\
\hline$L_{b}$ & 11.0 & {$[\mathrm{~mm}]$} \\
\hline$h$ at breakup & 19.9 & {$[\mu \mathrm{m}]$} \\
\hline$d_{L}$ & 87.7 & {$[\mu \mathrm{m}]$} \\
\hline$d_{r r}$ & 166 & {$[\mu \mathrm{m}]$} \\
\hline
\end{tabular}

\section{Euler-Lagrange approach}

Euler-Lagrange approach is used to model the dispersed phase in the air flow. The computational domain is as shown in Figure 5. The computational domain was obtained by considering the entire measuring section of the experimental test rig ( 1 meter) and the pipes before and after this section up to the experimental pressure ports (approximately 1 meter on each side). This avoided placing the inlet and outlet boundaries too close to the measuring section and at the same time allowed using experimental conditions in the numerical model.

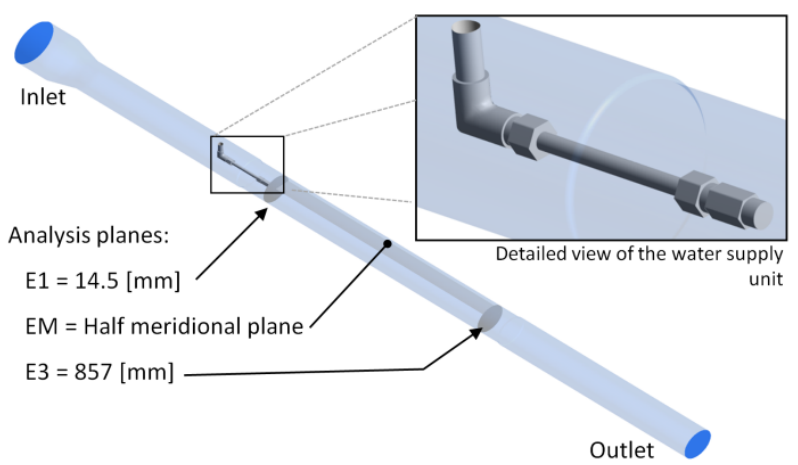

Figure 5: $\quad$ Euler-Lagrange computational domain.

Structured grid is used for the whole domain. Refinements are provided near the wall boundaries to obtain $y+\leq 40$. The grid statistics are presented in table 4. Continuity, momentum and energy equation are solved for the continuous phase. Turbulence is modelled using a two equation SST model. Mass flow and total temperature are set for the continuous phase inlet boundary condition, while average static pressure is set at the outlet.

The discrete phase is modelled considering the Newton laws accounting the drag forces to solve the particle dynamics. Heat transfer and evaporation are also 
modelled using default models available in ANSYS-CFX. Further details on these models could be found in ANSYS-CFX solver modelling guide [12].

Discrete phase boundary conditions include: particle diameter distribution, velocity and spray angle. The particle diameter distribution is described as a Rosin Rammler distribution using the reference diameter computed in the previous section. The exponent for the distribution is set to 3.3, which is obtained by curve fitted on to the experimental data. Velocity and spray angle are set according to the information obtained by the free surface model. At first the breakup point is identified and then the velocity and average flow direction at this point are used to define the spray initial conditions.

Table 4: $\quad$ Euler-Lagrange grid details.

\begin{tabular}{|l|c|l|}
\hline Euler Lagrange model & & \\
\hline Nodes & 4807124 & \\
\hline Elements & 4715912 & \\
\hline Near Wall Element height & 0.1 & {$[\mathrm{~mm}]$} \\
\hline
\end{tabular}

It is important to note that, strong differences in the velocity and spray angle could be seen between the nozzle exit and the breakup point. Figure 4 illustrates that the water sheet has a maximum angle of 26 [deg] at the nozzle exit, which reduces to 9 [deg] due to the drag imposed by the air on the water sheet at the point where breakup is expected.

For the presented case approximately 500,000 representative particles are used. The spatial discretization is second order accurate. After obtaining an approximate steady flow field for the continuous phase, the droplets are injected (approx. after 250 iterations) and tracked till they escape or reach a certain integration limit. The particle tracking is terminated when the water droplets hit the wall boundaries i.e. the perpendicular coefficient of restitution is set to zero. Iterations continue alternatively between the Eulerian and the Lagrangian phases. For every ten continuous phase iterations, one Lagrange iteration is performed. The Rhie-Chow algorithm is used for the pressure velocity coupling. The convergence criteria for a steady state simulation are met, when the RMS residuals for the mass, momentum, energy, mass fraction, particles source charges and turbulence fall below a value of $10 \mathrm{e}-4$. Approximately 500 to 600 iterations are needed to obtain converged results which take nearly 168 hours on a cluster with 10 cores.

\subsection{Results and discussion}

To present the calculations of multiphase flow in a stationary testrig, contour plots of Euler-Lagrange flow simulations are compared with the experimental droplet data obtained from a hot air test rig. Figure 6 illustrates the normalized mass flow distribution on half meridional plane of the measuring section. This mass flow is obtained by evaluating the mass crossing each measuring point in a unit time. The obtained values are then normalized in order to have better understanding of mass flow distribution. 
Comparing the normalized mass flow distributions on a half meridional plane illustrated in Figure 6 indicates that the main flow is limited to core region, specifically from $y=5[\mathrm{~mm}]$ to $y=25[\mathrm{~mm}]$ along the axis. In Figure 6 , experiments show that the flow starts converging towards the axis at approx. $\mathrm{x}=$ 200 [mm] which cannot be observed in CFD calculations. A possible reason for this could be due to the lost history of the primary breakup, which doesn't allow this phenomenon to develop in a Euler-Lagrange simulation.

Figure 7 illustrates the distribution of SMD on a half meridional plane. It can be observed that the numerical simulations are capable of describing the main flow area with reasonable accuracy. Also substantial deviations can also be observed in the higher and lower SMD regimes. In the outer periphery of the spray, numerical results overestimate the maximum SMD by $20[\mu \mathrm{m}]$.Though the calculated results are deviating from the experimental data by approximately $20[\mu \mathrm{m}]$, but are still successful in reproducing the trend of the spray evolution.
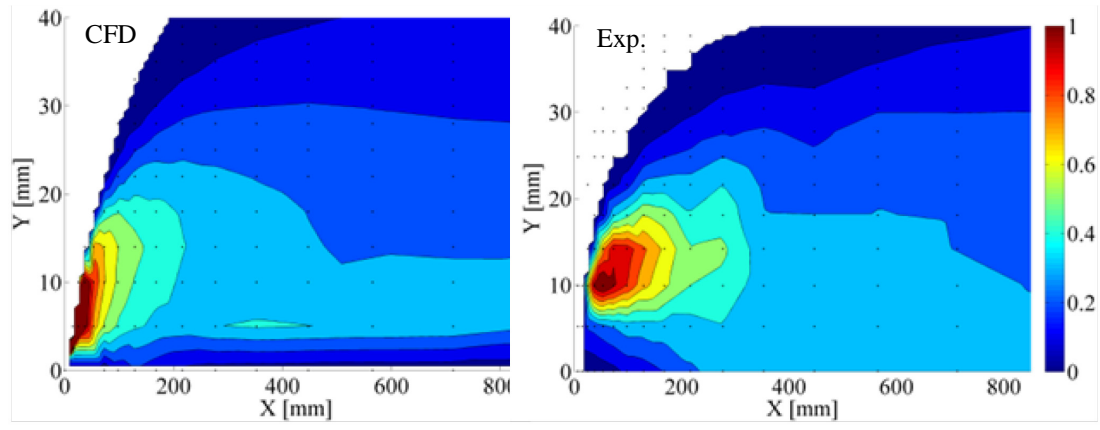

Figure 6: Normalized water mass flow distribution.
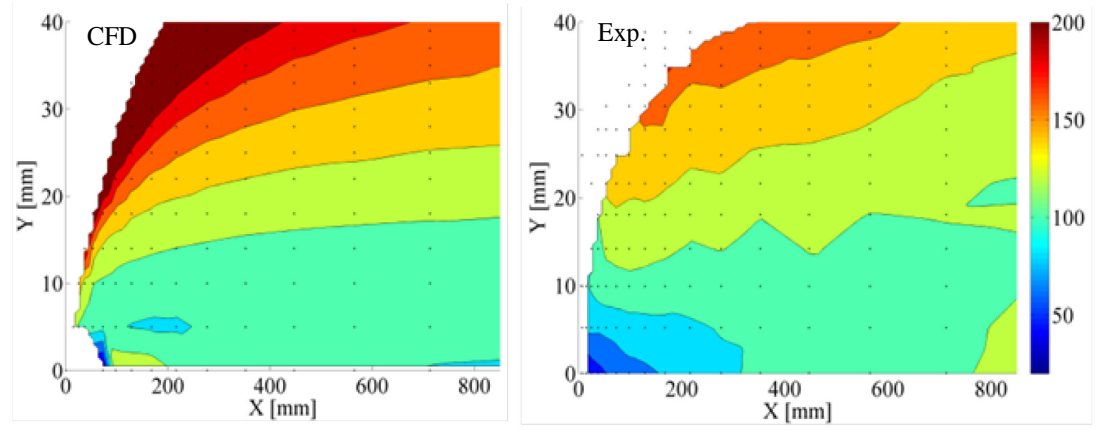

Figure 7: $\quad$ Sauter mean diameter distribution.

As illustrated in Figures 8 and 9, Lagrangian flow simulations are able to predict rather similar radial distribution of SMD and velocity, but not the nonuniform pattern observed in the experimental data. However, numerical data clearly shows the presence of the wake induced by an elbow of the water delivery pipe. The complex pattern observed in the experiments could be due to 
the presence of secondary flow inside the testrig at the inlet which is not accounted in this calculation.
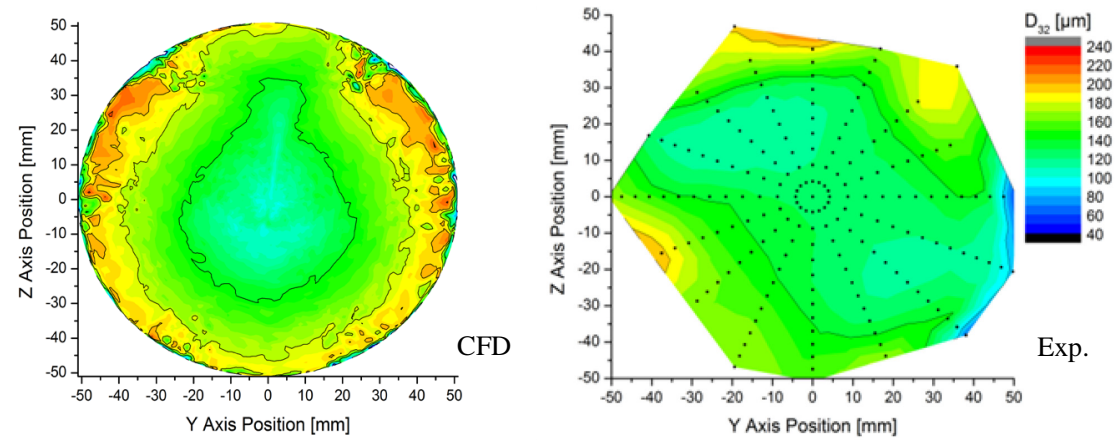

Figure 8: $\quad$ E3 cross section; Sauter mean distribution.
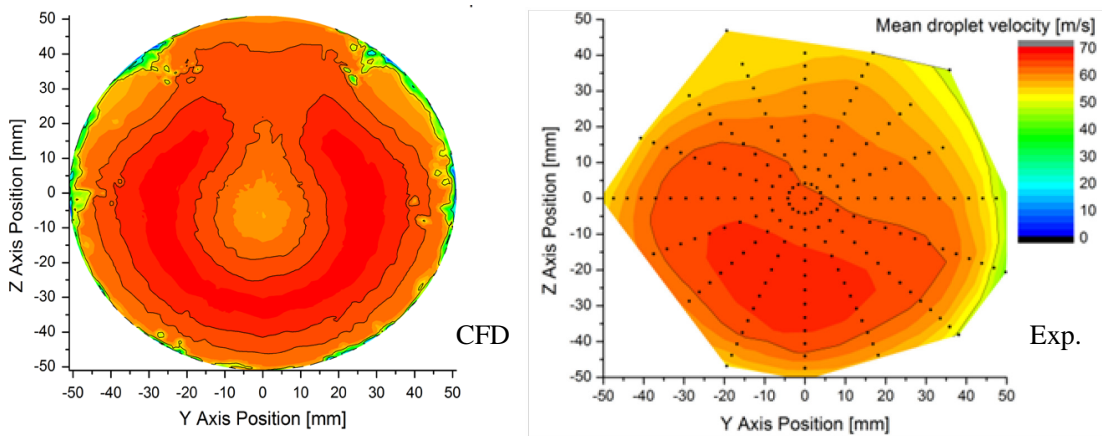

Figure 9: $\quad$ E3 cross section; particle velocity distribution.

\section{Conclusions}

One of the most critical point in spray simulations is the definition of the injection conditions for the Lagrangian phase, especially when the injection is performed at elevated temperature and pressure. In order to address this problem, this paper proposes a three stage approach to model the process of the water injection and breakup. A Euler two phase free surface flow analysis is first used to model the interaction between the upcoming high speed air flow and the water exiting the nozzle. The trajectory of the water sheet deformed by the interaction with the air flow is used to feed a Linearized Instability Sheet Atomization (LISA) model to predict the breakup. Euler-Lagrange analysis is then setup combining the information gathered by the previous models. Results showed the capability of the LISA model to predict the reference diameter of the particle at the nozzle exit with reasonable accuracy.

The comparison of the EL result with the experimental data showed a good agreement. The comparison of water mass flow distribution on the meridional plane allowed to confirm indirectly the values derived for the water injection 
velocity and direction. CFD also proved to be capable of predicting the SMD distribution on a half meridional plane with reasonable accuracy. Comparison of the experimental data on the radial plane E3 shown in Figure 5, suggested the presence of secondary flows inside the testrig, thereby promoting better mixing of the particles.

Further investigations using entire testrig has to be performed in order to assess the effects of secondary flow inside the testrig. Also the influence of turbulent dispersion of droplets and secondary breakup can be the subject for further investigations. However, in practice there can be many applications of water injection at elevated conditions. In such cases the presented approach can be usefully applied to study the primary breakup and droplet dynamics.

\section{Acknowledgement}

This project is funded by the German Federal Ministry of Economics and Technology. The authors are grateful for the financial support under contract number 0327861.

\section{Nomenclature}

$\begin{array}{lll}L & \text { Length } & \mathrm{m} \\ O h & \text { Ohnesorge number } & \\ Q & \text { Density ratio between gas and liquid } & - \\ T, \tau & \text { Time } & \mathrm{s} \\ U & \text { Liquid - gas relative velocity } & \mathrm{m} / \mathrm{s} \\ V & \text { Velocity } & \mathrm{m} / \mathrm{s} \\ K & \text { Most critical wave number } & \\ d & \text { Diameter } & \mu \mathrm{m} \\ h & \text { Sheet half thickness } & \mathrm{mm} \\ k & \text { Wave number } & - \\ r & \text { Radius } & \mathrm{m} \\ \Omega & \text { Most unstable disturbance } & - \\ \eta & \text { Wave amplitude } & - \\ \mu & \text { Dynamic viscosity } & \mathrm{Pa} \mathrm{s} \\ v & \text { Kinematic viscosity } & \mathrm{m} / \mathrm{s} \\ \rho & \text { Density } & \mathrm{kg} / \mathrm{m}^{3} \\ \sigma & \text { Surface tension coefficient } & \mathrm{N} / \mathrm{m} \\ \omega_{r} & \text { Wave amplitude grow rate } & \end{array}$

\section{Subscripts}

$O \quad$ Initial, Inlet, Nozzle exit, Reference

$b \quad$ Breakup, Critical

L Ligament

I Liquid

g Gas

$r r \quad$ Representative 


\section{References}

[1] Khan, J.R. and Wang, T., "3D Modeling for Wet-Compression in a Single Stage Compressor Including Liquid Particle Erosion,” in ASME Turbo Expo: Power for Land, Sea and Air, Glasgow, UK, 14th-18th Jun, 2010.

[2] Sun, L., Zheng, Q., Li, Y., Luo, M., Bahrgava, R.K., "On the behavior of water droplets when moving onto blade surface in a wet compression transonic compressor," Journal of Engineering for Gas Turbines and Power, vol. 133, no. 082001-1, Aug 2011.

[3] Jones, W.P. and Sheen, D.H., "A probability density function method for modelling liquid fuel sprays.," Flow, Turbulence and Combustion, vol. 63, pp. 379-394, 1999.

[4] Fritsching, U., Spray Simulation.: Cambridge University Press, 2004.

[5] Lefebvre, A.H., Atomization and Sprays. New York: Hemisphere, 1989.

[6] von Lavante, E., Maatje, U. and Albina, F.O., "Investigation of unsteady effects in pressure swirl atomizers," in Proc. ILASS-Europe, 2002.

[7] Buelow, P.E.O., Mao, C.P. and Smith, S., "Two-phase CFD modeling of a simplex atomizer," in Proc. ILASS-Americas, 2003, p. 09A3.

[8] Hansen, K.G., Madsen, J., Trinh, C.M. , Ibsen, C.H., Solberg, T. and Hjertager, B.H., "A computational and experimental study of the internal flow in a scaled pressure-swirl atomizer.," in Proc. ILASS-Europe, pp. 351356.

[9] Senecal, P.K., Schmidt, D.P., Nouar, I., Rutland, C.J., Reitz, R.D., and Corradin, M.L., "Modeling High-speed Viscous Liquid Sheet Atomization," Intl. Journal of Multiphase flow, no. 25, pp. 1073-1097, 1999.

[10] Schnitzler, J.P., Feng, J., Benra, F.K., Dohmen, H.J. and Werner, K., “Test Rig Design for Investigations of Water Droplet Evaporation at High Pressure and Temperature Levels," in 14th International Symposium on Transport Phenomena and Dynamics of Rotating Machinery, Honolulu, USA, 27th Feb-2nd March , 2012.

[11] Kefalas, A., Barabas, B., Schnitzler, J.P., Benra, F.K., Dohmen, H.J., "Water Droplet Evaporation at High Pressure and Temperature Levels Part I: Experimental Investigations of Spray Patterns at Varied Test Conditions," in 9th International Conference on Heat Transfer, Fluid Mechanics, and Thermodynamics, Malta, 16th-18th Jul, 2012.

[12] ANSYS CFX help files.

[13] Dombrowski, N. and Jones, W.R., "The Aerodynamic Instability and Disintegration of Viscous Liquid Sheets," Chemical Engineering Science, no. 18, pp. 203-214, 1963.

[14] Dombrowski, N. and Hooper, P.C., "The effect of ambient density on drop formation in sprays," Chemical Engineering Science, no. 17, pp. 291-305, 1962. 